\title{
Lesiones hepáticas sugestivas de angioma en pacientes con hepatopatía crónica
}

\author{
A. Repiso, R. Gómez Rodríguez, C. González de Frutos, T. de Artaza, J. J. Sánchez Ruano, M. J. Pérez \\ Grueso y J. L. Martínez Potenciano
}

Servicio de Aparato Digestivo. Hospital Virgen de la Salud. Toledo

\section{RESUMEN}

Objetivo: el objetivo de nuestro estudio fue valorar en nuestro medio las características clínicas, ecográficas y evolutivas de los pacientes con hepatopatía crónica y lesiones ecográficas sugestivas de angiomas hepáticos.

Material y métodos: estudio retrospectivo realizado entre los pacientes recogidos en la base de datos de la Unidad de Ecografías del Servicio de Aparato Digestivo entre enero de 2000 y junio de 2004. Incluimos en el estudio a pacientes que presentaban datos clínicos y/o analíticos compatibles con hepatopatía crónica de cualquier etiología y en los que la ecografía abdominal ponía de manifiesto la existencia de al menos una lesión hepática compatible con angioma. Se han recogido los datos epidemiológicos, clínicos, ecográficos y evolutivos de estos pacientes.

Resultados: durante el periodo de estudio se diagnosticaron 58 pacientes con hepatopatía crónica y lesiones hepáticas sugestivas de angioma, de los cuales trece presentaban datos clínicos, analíticos, ecográficos y/o histológicos compatibles con cirrosis hepática. Ecográficamente se trataban de lesiones menores de $10 \mathrm{~mm}$ en el 50\% de los pacientes y en la mayoría de los casos localizadas en lóbulo hepático derecho. Durante el periodo de medio de 35 meses (6 a 168 meses) se pudo comprobar cómo en dos pacientes (3\%) las lesiones inicialmente interpretadas como angiomas se trataban en realidad de lesiones malignas (un hepatocarcinoma y unas metástasis de adenocarcinoma vesicular). En ambos casos los pacientes eran cirróticos. Por tanto, en el 15\% de los pacientes cirróticos de nuestra serie se demostró la naturaleza maligna de las lesiones inicialmente interpretadas como angiomas.

Conclusiones: en pacientes con hepatopatía crónica, sobre todo en cirróticos, un porcentaje no despreciable de las lesiones ecográficas interpretadas inicialmente como angiomas se tratan realmente de lesiones malignas.

Palabras clave: Angioma. Hepatocarcinoma. Tumores hepáticos. Cirrosis hepática.

\begin{abstract}
Objective: the aim of this study was to evaluate in our healthcare area the clinical, ultrasonographic, and evolutionary features of patients with chronic liver disease and angioma-like liver lesions on ultrasonography.

Materials and methods: we conducted a retrospective study amongst patients seen at the Ultrasonography Unit, Gastroenterology Department between January 2000 and June 2004. Included in the study were patients that presented with clinical and/or laboratory complaints consistent with chronic liver disease of any etiology, and those in which abdominal ultrasounds revealed the existence of at least one angioma-like liver lesion. All relevant epidemiological, clinical, ultrasonographic, and evolutionary data were carefully collected and recorded.

Results: in the course of our study, 58 patients were diagnosed with chronic liver disease and angioma-like liver lesions, of which 13 showed clinical, laboratory, ultrasonographic, and/or histological signs of liver cirrhosis. In 50\% of patients these lesions were less than $10 \mathrm{~mm}$ in diameter, and in most cases were located in the right hepatic lobe. During an average follow-up period of 35 months (6-168 months) we verified that, in two patients, these lesions, initially interpreted as angiomas were in fact malignancies (one hepatocellular carcinoma and one metastatic adenocarcinoma of the gallbladder). In both cases, the patients were cirrhotic. Thus, our study revealed that $15 \%$ of lesions found in cirrhotic patients initially interpreted as angiomas were actually malignant.

Conclusions: our study revealed that, in patients with chronic liver disease, particularly in cirrhotic patients, a considerable percentage of ultrasonographic lesions originally interpreted as angiomas are in fact malignant tumors.
\end{abstract}

Key words: Amgioma. Hepatocellular carcinoma. Liver neoplasms. Liver cirrhosis.

Repiso A, Gómez Rodríguez R, González de Frutos C, de Artaza T, Sánchez Ruano JJ, Pérez Grueso MJ, Martínez Potenciano JL†. Lesiones hepáticas sugestivas de angioma en pacientes con hepatopatía crónica. Rev Esp Enferm Dig 2007; 99: $259-263$.

Recibido: $28-03-06$

Aceptado: 09-01-07.

Correspondencia: Alejandro Repiso Ortega. Servicio de Aparato Digestivo. Hospital Virgen de la Salud. Avda. Barber, 30.45004 Toledo. e-mail. arepiso@ sescam.jccm.es. 


\section{INTRODUCCIÓN}

El hemangioma representa el tumor hepático benigno más frecuente, pudiendo encontrase hasta en el 5\% de la población adulta (1). Ecográficamente su aspecto es variable. Sin embargo, la mayoría tienen un patrón ecográfico característico con forma redondeada, hiperecogénica, de bordes bien definidos y patrón de ecos homogéneo (2).

Los pacientes con hepatopatía crónica, y sobre todo aquellos en estadio cirrótico, representan un grupo de riesgo para desarrollar lesiones hepáticas malignas $(3,4)$. Concretamente el hepatocarcinoma se suele presentar ecográficamente como una lesión focal de tamaño variable, bien delimitada, de bordes irregulares y con mayor frecuencia heteroecogénica (2). En otras ocasiones, aparece con respecto al parénquima hepático como una lesión hipoecoica, isoecogénica o hiperecogénica en caso de existir un componente esteatósico importante, focos hemorrágicos o hipervascularización, en cuyo caso puede plantear problemas diagnósticos al ser indistinguibles ecográficamente del angioma hepático (5).

El objetivo de nuestro estudio fue valorar en nuestro medio las características clínicas, ecográficas y evolutivas de los pacientes con hepatopatía crónica y lesiones ecográficas sugestivas de angioma hepático.

\section{MATERIAL Y MÉTODOS}

\section{Población de estudio}

Estudio retrospectivo realizado en el Hospital "Virgen de la Salud" de Toledo entre los pacientes recogidos en la base de datos de la Unidad de Ecografías del Servicio de Aparato Digestivo entre enero de 2000 y junio de 2004.

Incluimos en el estudio a pacientes que presentasen datos clínicos y/o analíticos compatibles con hepatopatía crónica de cualquier etiología (alteración persistente de la bioquímica hepática de más de 6 meses de duración) y en los que la ecografía abdominal pusiera de manifiesto la existencia de al menos una lesión hepática compatible con angioma, definida como una lesión focal de características sólidas, hiperecogénica, redondeada y de bordes bien definidos (Fig. 1). Fueron excluidos los pacientes perdidos durante el seguimiento (tres pacientes) y aquellos con lesiones focales hiperecogénicas que por sus características ecográficas planteaban en la primera exploración el diagnóstico de lesión ocupante de espacio de naturaleza maligna como primera posibilidad.

\section{Estudio ecográfico}

Los equipos de ultrasonidos empleados fueron un ecógrafo Toshiba modelo SSH-140 y un Hytachi modelo EUB-6500, utilizando una sonda de multifrecuencia. En

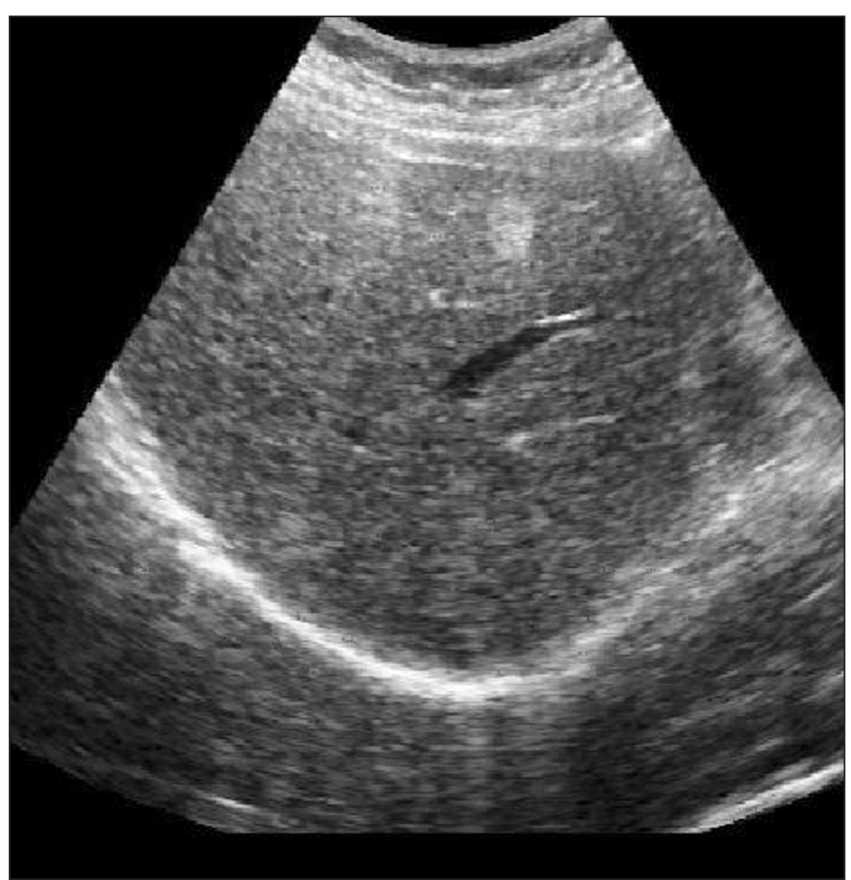

Fig. 1. Lesión hepática de bordes bien definidos, hiperecogénica, homogénea y redondeada, sugestiva de angioma.

la realización de los estudios participaron 4 exploradores con al menos 5 años de experiencia realizando ecografía abdominal, en la misma unidad de trabajo y con el mismo método.

\section{Seguimiento}

Los pacientes incluidos en el estudio eran seguidos en régimen ambulatorio, con visitas cada seis meses y ecografía abdominal cada seis o doce meses, en función de la etiología de la hepatopatía, estadio evolutivo y sospecha diagnóstica.

\section{Variables}

Las variables de valoración fueron:

1. Los datos clínicos incluidos fueron la edad del paciente al realizar la primera ecografía; la etiología de la hepatopatía; y la presencia cirrosis, definida por datos histológicos y/o clínicos, analíticos y ecográficos.

2. Datos ecográficos: el número, tamaño y localización de las lesiones (lóbulo hepático derecho y/o izquierdo); diagnóstico realizado en la primera ecografía o en exploraciones realizadas durante el seguimiento; existencia de datos ecográficos de cirrosis, definidos como alteración de la ecogenicidad del parénquima hepático, irregularidad de su superficie, hipertofia del lóbulo caudado y/o datos indirectos como ascitis o signos de hipertensión portal; la confirmación diagnóstica obtenida mediante el seguimiento ecográfico, o bien tras la realización de otras exploraciones 
complementarias (TAC y/o RMN). De modo general se decidió realizar TAC abdominal y/o RMN en aquellos pacientes en estadio cirrótico con lesiones focales hepáticas y/o en aquellos otros pacientes con lesiones hepáticas sin características típicas de angioma o cuando las características ecográficas se modificaban durante el seguimiento.

3. Datos evolutivos como el tiempo de seguimiento ecográfico; la diferencia de tamaño entre las lesiones observadas entre la primera y última ecografía; y la desaparición de alguna de las lesiones en los estudios ecográficos durante el periodo de seguimiento.

\section{RESULTADOS}

Entre los 1.950 pacientes incluidos en la base de datos con el diagnóstico de hepatopatía crónica, durante el periodo de estudio, en 58 (3\%) se observaron lesiones hepáticas sugestivas de angioma en las exploraciones ecográficas. Se trataba de 34 varones y 24 mujeres, con una media de edad de $52 \pm 12$ años, de los cuales 13 (22\%) presentaban datos clínicos, analíticos, ecográficos y/o histológicos de cirrosis hepática. La causa más frecuente de la hepatopatía crónica fue el virus de la hepatitis C (44\%) seguido por el virus de la hepatitis B (20\%), esteatohepatitis no alcohólica (7\%), hepatopatía etílica (4\%), hemocromatosis $(4 \%)$, hepatitis autoinmune $(2 \%)$, cirrosis biliar primaria (2\%) y otras causas (19\%).

En 39 pacientes se hizo el diagnóstico ecográfico de lesión hepática sugestiva de angioma en la primera exploración. Mientras que en 19 se observó por primera vez la lesión en alguna de las ecografías realizadas durante el periodo de seguimiento. La mitad de los pacientes presentaban lesiones menores de $10 \mathrm{~mm}$. En la mayoría de las ocasiones se trataban de lesiones únicas localizadas en lóbulo hepático derecho (Tabla I).

\begin{tabular}{ccc}
$\begin{array}{c}\text { Tabla I. Características ecográficas de } \\
\text { hepatopatía crónica y lesiones sugestivas de angioma }\end{array}$ & $\begin{array}{c}\text { Número de } \\
\text { pacientes }\end{array}$ & $\begin{array}{c}\text { Porcentaje } \\
\text { (\%) }\end{array}$ \\
\hline & 13 & 22 \\
\hline Datos ecográficos de cirrosis & 29 & 49 \\
Tamaño de las lesiones: < $10 \mathrm{~mm}$ & 11 & 19 \\
$>20 \mathrm{~mm}$ & 45 & 77 \\
Número de lesiones: Única & 13 & 22 \\
Múltiples & 45 & 77 \\
Localización: Lóbulo hepático derecho & 8 & 13 \\
Lóbulo hepático izquierdo & 5 & 8 \\
Ambos lóbulos & 39 & 67 \\
Diagnóstico: En la primera exploración & 19 & 32 \\
\hline
\end{tabular}

Para intentar aclarar el origen de la lesión se hizo TAC abdominal a 27 pacientes, consiguiéndose visualizar la lesión previamente descrita en 14 casos. Concretamente en los 11 pacientes con lesiones menores a $10 \mathrm{~mm}$ la TAC abdominal tan sólo consiguió visualizar las lesiones descritas ecográficamente en dos casos. En los 13 pacientes en estadio cirrótico se valoró la realización de TAC abdominal y/o RMN hepática que finalmente no se realizó en dos pacientes por su edad avanzada y mala situación clínica (Tabla II).

En el periodo de seguimiento medio de 35 meses $(6$ a 168 meses), en 56 pacientes el diagnóstico definitivo fue el de probable angioma hepático. En este periodo se observó variación en el tamaño de las lesiones (aumento o disminución del tamaño superior a $3 \mathrm{~mm}$ ) en 13 de los pacientes $(22 \%)$. En nueve disminución $(15 \%)$ y en cuatro aumento de tamaño (7\%). En todos los pacientes en los que se detectó aumento significativo del tamaño de las lesiones durante el seguimiento se decidió realizar

Tabla II. Características de los 13 pacientes cirróticos con lesiones hepáticas sugestivas de angioma

\begin{tabular}{|c|c|c|c|c|c|c|c|c|}
\hline Paciente & $\begin{array}{c}\text { Sexo } \\
*\end{array}$ & $\begin{array}{c}\text { Edad } \\
* *\end{array}$ & $\begin{array}{c}\text { Tamaño al } \\
\text { diagnóstico }(\mathrm{mm})\end{array}$ & $\begin{array}{c}\text { Seguimiento } \\
\text { ecográfico (meses) }\end{array}$ & TAC Abdominal & $R M N$ & Citología/histología & $\begin{array}{l}\text { Evolución y } \\
\text { tratamiento }\end{array}$ \\
\hline 1 & $M$ & 83 & 8 & 106 & No se hizo & No se hizo & No se hizo & Seguimiento \\
\hline 2 & $M$ & 76 & 10 & 11 & No se hizo & No se hizo & No se hizo & Exitus \\
\hline 3 & V & 81 & 5 & 20 & No LOES & No se hizo & No se hizo & Exitus \\
\hline 4 & V & 54 & 8 & 35 & No LOES & Hepatocarcinoma & Hepatocarcinoma & Resección; trasplante \\
\hline 5 & M & 45 & 6 & 3 & No LOES & No se hizo & Adenocarcinoma & Exitus \\
\hline 6 & V & 36 & 20 & 68 & LOE indeterminada & Angioma & No se hizo & Seguimiento \\
\hline 7 & V & 54 & 10 & 20 & LOE inespecífica & Angioma & No malignidad & Seguimiento \\
\hline 8 & V & 62 & 10 & 12 & LOE indeterminada & Angioma & No malignidad & Exitus \\
\hline 9 & V & 69 & 6 & 9 & LOE inespecífica & No LOES & No se hizo & Seguimiento \\
\hline 10 & V & 62 & 8 & 34 & No LOES & Angioma & No se hizo & Seguimiento \\
\hline 11 & M & 58 & 15 & 88 & No se hizo & No se hizo & Angioma & Trasplante \\
\hline 12 & V & 60 & 10 & 36 & Angioma & Angioma & No se hizo & Seguimiento \\
\hline 13 & V & 70 & 15 & 12 & No LOES & Angioma & Angioma & Trasplante \\
\hline
\end{tabular}

* M: mujer; V: varón; ** edad en el momento del diagnóstico. 
TAC abdominal (4 pacientes) y/o RMN hepática (un paciente). En algún momento del seguimiento, en 20 pacientes no se detectaron ecográficamente las lesiones descritas en exploraciones previas (Fig. 2).

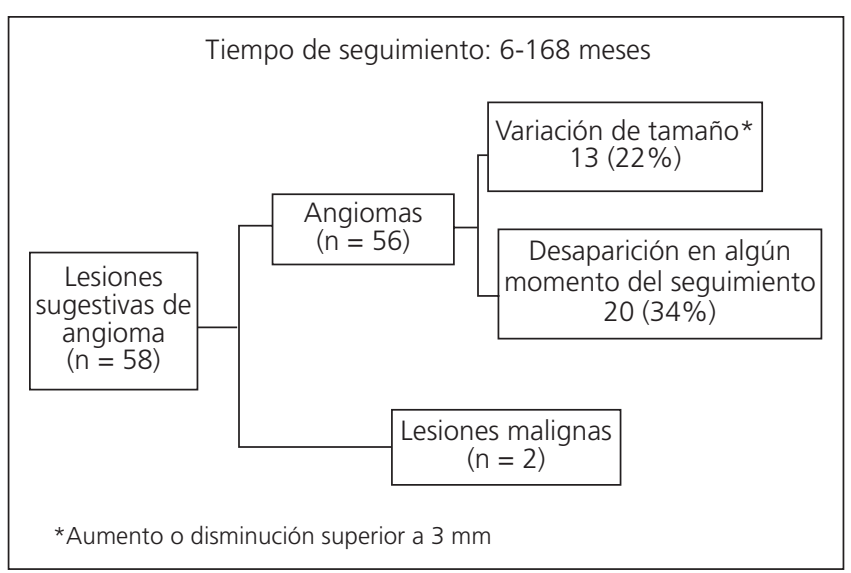

Fig. 2. Características evolutivas de 58 pacientes con hepatopatía crónica y lesiones sugestivas de angioma.

En dos enfermos (3\%) se pudo comprobar cómo las lesiones inicialmente descritas como angiomas se trataban en realidad de lesiones malignas. Concretamente en un paciente tras 35 meses de seguimiento se pudo demostrar histológicamente, tras modificarse las características ecográficas de la lesión, como se trataba de un hepatocarcinoma (Figs. 3a y 3b) y en otra paciente, de metástasis de un adenocarcioma vesicular. En ambos casos los pacientes presentaban datos clínicos, analíticos y/o ecográficos de cirrosis (Tabla II). Por tanto, en el 15\% de los pacientes cirróticos de nuestra serie se demostró la naturaleza maligna de las lesiones inicialmente interpretadas como angiomas.

\section{DISCUSIÓN}

Los pacientes con hepatopatía crónica representan un grupo de alto riego para desarrollar lesiones hepáticas malignas que en ocasiones pueden presentar un aspecto ecográfico indistinguible del angioma hepático. En nuestro medio, en el 3\% de los pacientes con hepatopatía crónica y lesiones inicialmente descritas como angiomas pudimos demostrar su origen maligno. Concretamente son los pacientes cirróticos los que tienen un mayor riesgo de presentar lesiones hepáticas malignas. Como ocurrió en nuestra serie, en los dos enfermos en que se pudo comprobar que las lesiones eran realmente malignas, se trataban de pacientes cirróticos. Por tanto, en el $15 \%$ de los pacientes cirróticos de nuestra serie se demostró la naturaleza maligna de las lesiones inicialmente interpretadas como angiomas. En otras series se llega a demostrar
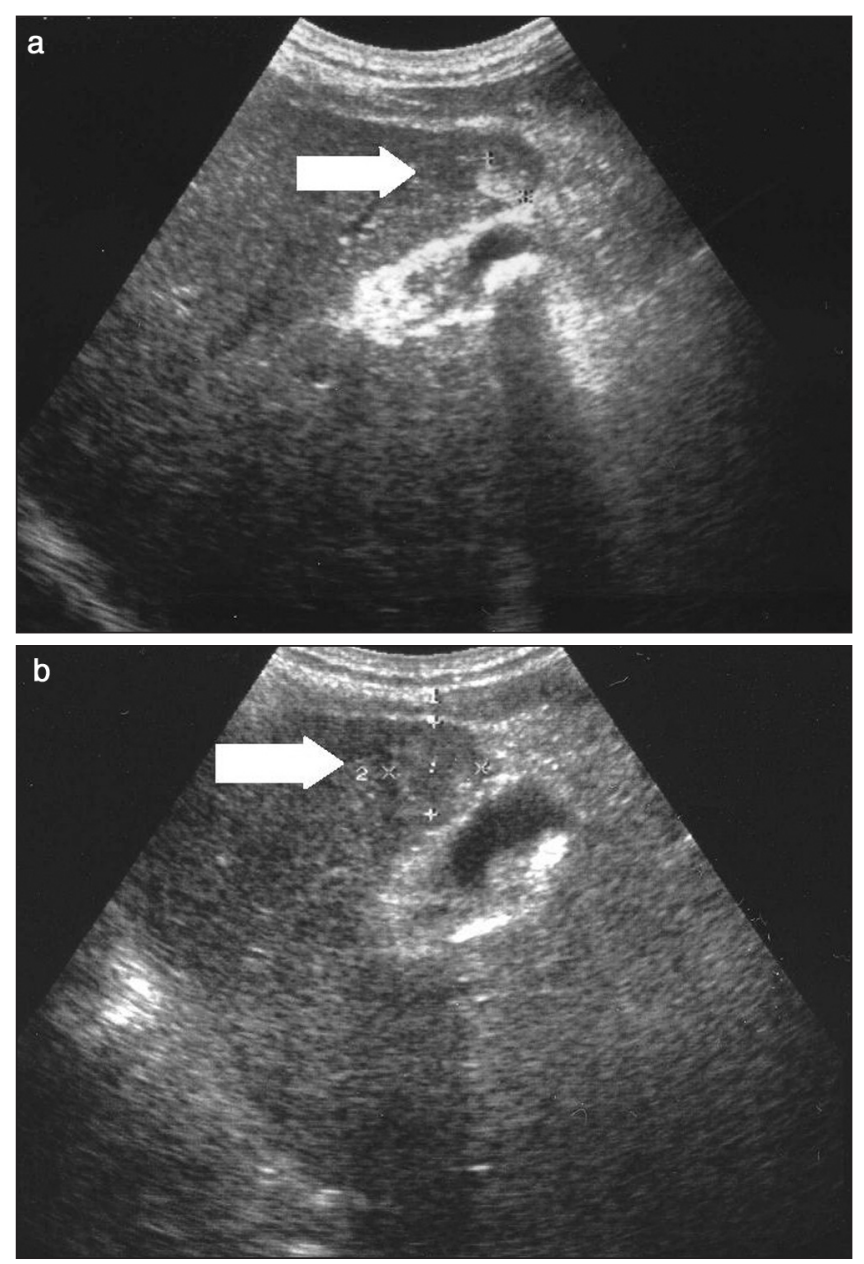

Fig. 3. a. Lesión de $8 \mathrm{~mm}$ sugestiva de angioma (flecha), localizada en lóbulo hepático izquierdo en paciente con cirrosis hepática. b. Trascurridos 35 meses de la primera exploración se aprecian cambios en las características de la lesión descrita previamente, observándose una lesión de mayor tamaño, isoecogénica respecto al paréquima hepático, con halo periférico hipoecogénico, sugestiva de hepatocarcinoma (flecha).

cómo hasta el 50\% de estas lesiones sugestivas de angiomas en pacientes cirróticos se tratan de hepatocarcinomas hiperecogénicos o lesiones preneoplásicas (5).

Generalmente, se acepta que los pacientes de bajo riesgo con lesiones focales hepáticas detectadas en ecografía y con datos típicos de angioma no precisan más exploraciones complementarias (6). Sin embargo, en los pacientes con cirrosis hepática, al tratarse de un grupo de alto riesgo, parece recomendable la realización de otras técnicas de imagen para confirmar el diagnóstico (4). La elección de una determinada técnica quedará influenciada por la experiencia y disponibilidad en cada centro. A pesar del alto rendimiento diagnóstico de la TAC abdominal referido en el diagnóstico de lesiones focales hepáticas $(7,8)$, en nuestro medio, resultó ser una técnica poco sensible en esta indicación, probablemente por la alta pro- 
porción de lesiones de pequeño tamaño $(<1 \mathrm{~cm})$ observadas ecográficamente, lo cual limita el poder de resolución de esta técnica, como se ha referido en otros trabajos (3,4,9-11). El reciente desarrollo de los contrastes ecográficos permitirá mejorar la caracterización de este tipo de lesiones hepáticas y realizar un diagnóstico diferencial ecográfico más preciso (12-14).

En cuatro de los trece pacientes cirróticos se realizó punción guiada por ecografía de la lesión para obtener un diagnóstico citológico. Esta técnica es segura y rentable, incluso en el diagnóstico de lesiones de pequeño tamaño $(15,16)$, pero con frecuencia en estos casos un resultado negativo no permite descartar el diagnóstico de malignidad $(3,4)$. Por lo que en nueve pacientes cirróticos se decidió no realizar estudio citológico, como aquellos de edad avanzada, en caso de negativa del paciente o en aquellas lesiones de pequeño tamaño menores de $10 \mathrm{~mm}$, en cuyo caso se decidió realizar seguimiento ecográfico trimestral $(3,4,9)$. Tradicionalmente se han considerado los angiomas lesiones no susceptibles de punción. Sin embargo, resulta un procedimiento seguro y recomendable en pacientes con imagen atípica y/o riego elevado de neoplasia maligna $(17,18)$.

El riesgo de tratarse de una lesión maligna parece ser mayor cuando la lesión sugestiva de angioma se detecta durante el seguimiento o en los casos en que se modifican sus características ecográficas (5). Especial atención merecen las lesiones que aumentan de tamaño, pues parece poco probable que un tumor de naturaleza congénita, como es el angioma hepático, aumente de tamaño de forma significativa o lo suficiente como para poder ser detectado ecográficamente.

La disminución o desaparición de los angiomas durante el seguimiento en los pacientes con hepatopatía crónica es un hecho conocido (19) que se ha relacionado, no sólo con factores dependientes del explorador, sino también con cambios en la ecogenicidad del parénquima hepático y de las propias lesiones, debido al aumento del contenido fibrótico, que dificultan su identificación (19). Pero también puede ser un dato que oriente a la naturaleza maligna de las lesiones, como es el caso de uno de nuestros pacientes, en el que tras 35 meses de seguimiento de una lesión menor de $10 \mathrm{~mm}$, desapareció en uno de los controles ecográficos trimestrales, presentándose posteriormente en la misma localización como una lesión de mayor tamaño y sugestiva de hepatocarcinoma.

En conclusión, en pacientes con hepatopatía crónica y sobre todo en enfermos cirróticos un porcentaje no despreciable de las lesiones ecográficas interpretadas inicialmente como angiomas se tratan realmente de lesiones malignas. Por lo tanto, parece recomendable en estos en- fermos el seguimiento ecográfico estrecho y la realización de estudios complementarios, sobre todo en los pacientes cirróticos y en los que existen modificaciones de las características ecográficas durante el seguimiento.

\section{BIBLIOGRAFÍA}

1. Ishak KG, Rabin L. Benign tumors of the liver. Med Clin North Am 1975; 59: 995-1013.

2. Gómez-Rodríguez RA, Sánchez-Ruano JJ, Martínez-Potenciano JL. Ecografía en la patología hepática. En: Segura-Cabral JM, editor. Ecografía abdominal. $2^{\mathrm{a}}$ ed. Madrid: Ediciones Norma S.L.; 1996. p. 27-102.

3. Sala M, Llovet JM, Bruix J. Consideraciones acerca de la Conferencia de Barcelona sobre diagnóstico y tratamiento del carcinoma hepatocelular. Gastroenterol Hepatol 2002; 25: 570-4.

4. Bruix J, Sherman M; Practice Guidelines Committee, American Assocition for the Study of Liver Diseases. Management of hepatocellular carcinoma. Hepatology 2005; 42: 1208-36.

5. Caturelli E, Pompili M, Bartoluccci F, Siena DA, Sperandeo M, Andriulli A, et al. Hemangioma-like lesions in chronic liver disease: diagnostic evaluation in patients. Radiology 2001; 220: 337-42.

6. Leifer DM, Middleton WD, Teefey SA, Menias CO, Leahy JR. Follow-up of patients at low risk for hepatic malignancy with characteristic hemangioma at US. Radiology 2000; 214: 167-72.

7. Freeny PC, Marks WM. Hepatic hemangioma: dynamic bolus computed tomography. AJR 1986; 147: 711-9.

8. Quinn SF, Benjamin GG. Hepatic cavernous hemangiomas: simple diagnostic sign with dynamic bolus CT. Radiology 1992; 182: 545-8.

9. Bruix J, Sherman M. Diagnosis of small HCC. Gastroenterology 2005; 129: 1364

10. Horigome H, Nombra T, Saso K, Itoh M, Joh T, Ohara H. Limitations of imaging diagnosis for small hepatocellular carcinoma: comparison with histological findings. J Gastroenterol Hepatol 1999; 14: 559-65.

11. Kanematsu M, Hoshi H, Yamada T, Murakami T, Kim T, Kato M, et al. Small hepatic nodules in cirrhosis: ultrasonographic, CT, and MR imaging findings. Abdom Imaging 1999; 24: 47-55.

12. Isozaki T, Numata K, Kida T, Hara K, Morimoto M, Sakaguchi T, et al. Differential diagnosis of hepatic tumors by using contrast enhancement patterns at US. Radiology 2003; 229: 798-805.

13. Quaia E, Calliada F, Bertolotto M, Rossi S, Garioni L, Rosa L, et al Characterization of focal liver lesions with contrast-specific US modes and a sulfur hexafluoride-filled microbubble contrast agent: Diagnostic confidence. Radiology 2004; 232: 420-30.

14. Gómez R, Artaza T, González C, Repiso A, Sánchez JJ, Pérez MJ, et al. Valor del estudio con contrastes ecográficos de segunda generación en LOEs detectadas en pacientes con hepatopatía. Rev Esp Enferm Dig 2005; 97: A39.

15. Fornari F, Filice C, Rapaccini GL, et al. Small $(<3 \mathrm{~cm})$ hepatic lesions: results of sonographically guided fine-needle biopsy in 385 patients. Dig Dis Sci 1994; 39: 2267-75.

16. Yu S, Liew C, Lau WY, Leung TW, Metreweli C. US-guided percutaneous biopsy of small $(<1 \mathrm{~cm})$ hepatic lesions. Radiology 2001; 218: 195-9.

17. Gil-Ares F, Gómez-Rubio M, Santonja-Garriga C, De Cuenca-Morón B, García-Albarez J. Punción-biopsia diagnóstica en el hemangioma hepático. Rev Esp Ecogr Dig 2003; 5: A137.

18. Tung GA, Cronan JJ. Percutaneous leedle biopsy of hepatic cavernous hemangioma. J Clin Gastroenterol 1993; 16: 117-22.

19. Brancatelli G, Federle MP, Blachar A, Grazioli L. Hemangioma in the cirrhotic liver: Diagnosis and natural history. Radiology 2001; 219: 69-74 